\title{
Perspectivas do ensino de Geociências
}

\author{
MARCIA ERNESTO, ${ }^{I}$ \\ UMBERTO G. CORDANI, II \\ CELSO DAL RÉ CARNEIRO, ${ }^{I I I}$ \\ MARIA ASSUNÇÃO F. DA SILVA DIAS, ${ }^{I V}$ \\ CARLOS ALBERTO MENDONÇA ${ }^{V}$ \\ - ELISABETE DE SANTIS BRAGA VI
}

\section{Contexto histórico}

$\mathrm{O}$

ENSINO de Ciências foi incorporado aos currículos escolares somente no século XIX. Até então, focava-se no ensino de línguas clássicas e matemática. A forma de se entender as Ciências Naturais estava, até então, muito condicionada às tradições e à forma como se via o mundo que nos rodeia. Entender as leis da Natureza era o caminho para "dominar" a Terra e explotar os recursos disponíveis. Assim, as ciências eram vistas de forma totalmente utilitarista. A Revolução Industrial levou ao reconhecimento de que a ciência e a tecnologia tinham o poder de transformar a economia das sociedades, o que levou a formalizar o ensino da Física, Química e Geologia, com a preocupação de formar indivíduos para ensinar essas matérias.

Duas correntes de pensamento reinavam na época: a corrente que podemos chamar de "utilitarista", segundo a qual a ciência tinha um sentido mais prático e que deveria ajudar na resolução dos problemas cotidianos, e aquela da ciência acadêmica voltada à formação de cientistas. Krasilchik (2000) aponta a década de 1950 como o marco para a transformação do ensino da Ciência e Tecnologia, crescendo em todos os níveis, à medida que se reconhecia o poder transformador das ciências no desenvolvimento econômico, cultural e social das nações. De fato, as Ciências da Natureza de que tratamos aqui, Geologia, Geofísica, Meteorologia e Oceanografia, tiveram seus primeiros cursos de nível superior criados nessa época (ver Tabela 1, p.317-18, do artigo "Ensino de Geociências na universidade" de Cordani et al., neste volume).

No Brasil Império o ensino das Ciências Naturais era incipiente, embora, desde a criação do Colégio Pedro II, em 1837, as ciências já fizessem parte do currículo do ensino secundário. Todavia, alguns requisitos essenciais para o bom ensino de Ciências eram ausentes, tais como a ausência de atividades experimentais (Chassot, 1996). 
Assim como os estudos de campo permitem reconhecer a geologia do ambiente em que se vive, as atividades observacionais orientam o olhar do aluno para questões ambientais, colaboram para o entendimento dos mecanismos que governam a dinâmica terrestre e ajudam a desenvolver o pensamento crítico. A perspectiva sistêmica de Ciência do Sistema Terra (CST) estabelece novos modos de entendimento dos processos interativos que compõem a dinâmica planetária e as múltiplas relações entre geosfera, hidrosfera (que inclui a criosfera, sólida), atmosfera, biosfera e antroposfera (Carneiro; Gonçalves, 2010) e ajuda a construir uma visão aprimorada do passado, do presente e do futuro da Terra (Drummond; Markin, 2008). As atividades humanas são objeto de estudo de CST, uma vez que a biosfera e a antroposfera estão incluídas. A visão sistêmica das Ciências da Terra é, enfim, importante ponto de convergência para integrar os conhecimentos de física, matemática, química e biologia e auxiliar o aluno a construir o conhecimento. Assim, a oceanografia, como ciência que procura observar e explicar os fenômenos físicos, químicos, geológicos e biológicos que se processam nos oceanos, possui forte inter-relação com a geofísica e a meteorologia. Tais ciências, ligadas à tecnologia e à Engenharia Oceânica (Galo; Verrone, 1993), apresentam abordagem multidisciplinar, com alto potencial para colaborar em temas ambientais. Consequentemente, as Ciências da Terra podem servir como uma introdução à vida e às ciências físicas ou como um curso fundamental. Os conteúdos que desenvolvem, entretanto, aparecem de maneira incipiente no conteúdo curricular da escola básica.

No Brasil, a Lei n.4.024 - Diretrizes e Bases da Educação, de 21 de dezembro de 1961, ampliou a participação das ciências no currículo escolar, desde o $1^{\circ}$ ano do curso ginasial e com substancial aumento da carga horária de Física, Química e Biologia, no Segundo Grau. Nova alteração da lei em 1971, a Lei de Diretrizes e Bases da Educação n.5692, deu ao então Segundo Grau um caráter pretensamente mais profissionalizante, e às Ciências Naturais, um caráter mais instrumental (Krasilchik, 2000). Em 1996, uma nova Lei de Diretrizes e Bases da Educação (Lei n.9.394/96) estabelece no artigo 26 que "os currículos do ensino fundamental e médio devem ter uma base nacional comum, a ser complementada pelos demais conteúdos curriculares especificados nesta Lei e em cada sistema de ensino". Aqui também, o ensino de Ciências deveria ser voltado à preparação do indivíduo para o trabalho.

A reestruturação da Base Nacional Comum Curricular (BNCC) para o Ensino Médio, a partir da Lei n.13.415/2017, pretende atualizar e flexibilizar o currículo dessa fase do ensino, facultando ao estudante fazer suas escolhas curriculares e, assim, mantê-lo mais tempo na escola e de modo mais motivado. A BNCC definirá as competências e objetivos de aprendizagem nas quatro áreas do conhecimento: linguagens e suas tecnologias, matemática e suas tecnologias, ciências da natureza e suas tecnologias e ciências humanas e sociais aplicadas. Acredita-se que a medida ajudará a manter na escola um grande contingente (cerca de $25 \%$ ) de jovens que abandonam o Ensino Médio por falta de interesse. 
Em outras palavras, se a escola oferecer todas as opções, o jovem poderá escolher em que se especializar, podendo concentrar $40 \%$ da carga horária em disciplinas dessas áreas. $\mathrm{O}$ ensino de Português e de Matemática continuará sendo obrigatório nos três anos do Ensino Médio.

Embora a alteração possa trazer alguns benefícios, veem-se com preocupação tanto as defasagens na formação de diferentes grupos de jovens quanto a lacuna que poderá existir em relação a princípios científicos básicos e aqueles que se referem às Ciências da Terra em particular. A complexidade dos problemas naturais requer o entendimento dos fenômenos físicos que ocorrem na Natureza e como eles se manifestam no ambiente que habitamos. A Sociedade Geológica Americana, por exemplo, compreende que o conhecimento básico sobre as Ciências da Terra é essencial para atender os desafios ambientais e limitações de recursos naturais do século XXI.

É fundamental que a educação ambiental, e, mais amplamente, a educação em Geociências, comece nos níveis mais fundamentais do ensino e avance para conhecimentos mais aprofundados na escola secundária. Tomemos por exemplo a polêmica sobre aquecimento global antropogênico, que tem sido debatida na atualidade. Em que pese a realidade dos indicadores ambientais atuais, também é interessante instruir a população sobre os ciclos naturais que afetam o clima na escala do tempo geológico:

[...] a variabilidade natural do clima recente consiste na sobreposição de várias oscilações, muitas de origem astronômica, que vão desde a escala decenal até a escala milenar (Scafetta, 2014).

Tópicos desafiadores e pluridisciplinares como esse certamente não podem ser assimilados por um público carente de formação escolar multidisciplinar nas diversas áreas do conhecimento. O ensino de Geociências atualmente é invisível nos currículos escolares. Há tópicos inseridos na disciplina de Geografia, porém a dinâmica do planeta e as consequências das intervenções antropogências precisam ser ministradas de forma mais sistemática, para que haja um entendimento mais completo dos mecanismos que levam às transformações do meio ambiente. A situação se complica mais ainda quando se consideram os quadros técnicos de governo, que são muitas vezes levados a tomar decisões sem dispor de uma base de conhecimento adequado. É, portanto, imprescindível que haja base de conhecimentos sólidos e atualização técnico-científica das equipes responsáveis pelo enfrentamento das questões ambientais.

A comparação do nível de aprendizado em Ciências no Brasil com relação ao mundo pode ser visto nas avaliações do PISA (Programa Internacional de Ensino) da OCDE (Organização para a Cooperação e Desenvolvimento Econômico) reproduzidas pelo INEP. ${ }^{1} \mathrm{Na}$ avaliação do aprendizado dos estudantes do ensino médio em Matemática e Ciências o Brasil aparece abaixo da média dos países avaliados. Este é portanto um tema de profunda relevância para o país já que o desenvolvimento econômico dos países mais bem colocados nessa 
avaliação é reconhecidamente baseado em investimentos e atuação em Ciência e Tecnologia.

\section{Mercado de trabalho e necessidades nacionais e mundiais}

Ao se considerar projeções de atuação profissional e do mercado de trabalho, necessariamente deve-se considerar o perfil multidisciplinar fornecido pelos cursos em Geociências e a existência de um campo ainda a ser explorado na área de serviços. O perfil multidisciplinar desses profissionais cria habilitações para interfaces com diversas áreas da ciência e das tecnologias que envolvem o uso de instrumentação científica e quantificação utilizando recursos tecnológicos e computacionais. A capacidade de "interfaceamento", no sentido de identificar novos campos de atuação, deve ser estimulada nos cursos de graduação e pós-graduação. $\mathrm{O}$ quadro exige a abertura de espaço criativo para transformar resultados de pesquisa, na maioria das vezes provenientes de estudos estritamente acadêmicos, em abordagens que possam atender demandas do setor produtivo.

No caso da geologia e da geofísica, o Brasil, com o seu território de dimensões continentais, oferece amplas oportunidades de trabalho. Além dos trabalhos para o próprio conhecimento do território, o campo de atuação em que geólogos e geofísicos são parceiros é o da exploração mineral. Isso ocorre tanto no reconhecimento geológico de grande escala e levantamentos arogeofísicos como em trabalhos de prospecção realizados localmente na vizinhança de áreas em que foram identificadas ocorrências minerais. No caso da plataforma continental, a combinação de dados geofísicos com os dados de sondagens ajuda na busca de jazimentos de petróleo e gás. Em outras áreas, geólogos e geofísicos atuam como consultores ou chefiando empresas de serviços, como é o caso de gerenciamento de águas subterrâneas, de auxilio complementar em grandes obras de engenharia civil, ou de assessoramento em estudos e pesquisas ambientais. A capacidade de "interfaceamento" de geólogos e geofísicos com outras áreas da ciência, no sentido de identificar novos campos de atuação, deve ser estimulada em seus cursos de graduação e pós-graduação. $\mathrm{O}$ quadro exige a abertura de espaço criativo para transformar resultados de pesquisa, na maioria das vezes resultantes de estudos estritamente acadêmicos, em abordagens que possam atender demandas do setor produtivo.

A oceanografia também apresenta acentuado aspecto multi- e interdisciplinar em suas abordagens físicas, químicas, geológicas e biológicas ao formar o profissional com conhecimento de seu principal objeto de estudo, os oceanos e mares costeiros, ligados aos fenômenos de média e larga escalas associados às mudanças globais e climáticas e aos recursos vivos e não vivos. Porém, deve ser feito investimento na ampliação do leque de formação associado aos sistemas de interface como os estuários e os sistemas límnicos, nos quais as notáveis alterações antrópicas são interfaceadas com os oceanos, demandando ações profissionais para a preservação, sustentabilidade, manejo e gestão de recursos hídricos e marinhos. Também notável tem sido a incorporação do espírito da investigação 
científica no ensino de graduação em projetos de Iniciação Científica e de conclusão de curso, os quais envolvem atividades de formação em campo, com a necessidade de trabalhos práticos, colocando o futuro profissional em contato direto com seu meio de atuação via observações astronômicas e meteorológicas, excursões terrestres e embarques.

A meteorologia e, de uma forma mais geral, as ciências atmosféricas apresentam-se cada vez mais como atividades multidisciplinares fortemente baseadas em tecnologia e inovação. $\mathrm{O}$ mercado de trabalho se expande e incorpora os organismos governamentais tradicionais e, de uma forma surpreendente, a iniciativa privada. Conceitos e técnicas antes restritos à academia como modelagem numérica da atmosfera e métodos de abordagem de BigData cada vez mais sofisticados passam a ser uma demanda das novas empresas que atendem setores produtivos na agricultura de precisão, energia hidrelétrica, eólica e solar. Aspectos ambientais como a poluição de ar abordam cada vez mais tecnologia de observação e os órgãos de controle ambiental se modernizam e agregam profissionais com formação interdisciplinar em meteorologia e química da atmosfera. A formação do profissional tem nesse cenário um duplo desafio de abordar aspectos básicos da meteorologia ao mesmo tempo que sofisticadas técnicas de observação (especialmente de sensoriamento remoto com satélites e radares) e tratamento de dados. O objetivo é incorporar no ensino de graduação a noção de que o aprendizado de novas técnicas será uma constante em sua vida profissional.

Certamente, o maior desafio que se coloca à graduação é conectar esse conhecimento ao setor produtivo e, com isso, contrapor (ou, ao menos, amenizar) as flutuações do mercado de trabalho tradicional (principalmente, petróleo e gás). A criação de novos nichos de atuação implica incorporar como tecnologia os conhecimentos advindos da pesquisa de pós-graduação. Não necessariamente os setores tradicionais de emprego devam ser desconsiderados, mas novos espaços podem ser criados com base na qualificação dos egressos.

A sociedade moderna, em constante transformação, exige respostas rápidas dos currículos de terceiro grau para atender às demandas do país em termos de tecnologia, inovação, gestão e sustentabilidade. Desde as últimas décadas do século XX estamos enfrentando desafios enormes em todos os setores sociais e econômicos, com os avanços na microeletrônica, informática, biotecnologia, genética e em tantas outras ciências, implicando profundas transformações do setor produtivo. O processo de globalização, ao contrário do que o nome pode sugerir, não é inclusivo; ao contrário, os benefícios da nova ordem econômica concentram-se nos países com maior poder de deter tecnologias. O desenvolvimento e/ou absorção de novas tecnologias depende do preparo escolar que uma sociedade pode oferecer aos cidadãos.

$\mathrm{Na}$ segunda metade do século XX, ao mesmo tempo que a Terceira Revolução Cientifica e Tecnológica, como denominado por Libâneo et al. (2012), 
trouxe progresso e bem-estar sociais, o meio ambiente se viu agredido e ameaçado pela busca cada vez mais acentuada de insumos econômicos e de subsistência e pelos efeitos negativos dessas práticas - contaminação ambiental e explotação irresponsável, comprometendo gerações futuras. A consciência mundial em relação à essa degradação ambiental foi despertada nos anos 1950-1960, em que o planeta contava com uma população de cerca de 2,5 bilhões de pessoas. O termo "ecodesenvolvimento" apareceu na conferência mundial de Estocolmo, em 1972, e o movimento em defesa do ambiente cresceu na década de 1980 com a Comissão Bruntland, que criou a expressão "desenvolvimento sustentável" em seu livro Nosso futuro comum. Na maior conferência que as Nações Unidas já organizaram, a Rio-92, o documento principal, a Agenda-21, deixou claro que os modelos socioeconômicos vigentes não seriam sustentáveis, e indicou as muitas ações que deveriam ser feitas para um futuro sustentável, como agricultura regenerativa, reciclagem industrial e tecnologias "verdes", entre outras. Os primeiros economistas a indicar que os avanços tecnológicos seriam a única saída para evitar o colapso do planeta por causa da explosão populacional foram Enrich e Holdren (1971). Esses autores sugeriram que o impacto ambiental seria uma função do crescimento populacional, consumo per capita e desenvolvimento tecnológico. Presentemente há no mundo 7,5 bilhões de pessoas, três vezes mais do que em 1950, e o crescimento continua. O consumo unitário também deverá aumentar, acompanhando o desenvolvimento socioeconômico. Dessa forma, a tecnologia, operando do modo indicado pela Agenda 21, é o que restaria para manter o impacto ambiental abaixo das condições de suporte do planeta, favorecendo a restauração ambiental.

A questão ambiental mudou o mundo. Preservação e conservação ambiental viraram cultura popular, os programas de ensino formais do mundo inteiro acompanharam essa evolução e o ambiente veio a permear a educação das populações. Pelo conhecimento que têm a respeito do planeta e de seus recursos, as Geociências foram consideradas de grande relevância. Os profissionais das Geociências transformaram-se em guardiões do planeta e seu papel é fundamental no monitoramento das mudanças climáticas, na mitigação dos desastres naturais, na busca e gerenciamento de recursos minerais energéticos e hídricos, e na proteção dos solos agrícolas (Cordani, 1998). Com relação às mudanças climáticas e ao aquecimento global, devido às emissões de gás carbônico antropogênico, que se faz sentir desde a Revolução Industrial do século XIX, o panorama é sombrio. As Geociências estão no centro desse grande debate e têm muito a contribuir, tanto no estudo e compreensão dos fenômenos quanto na prevenção e mitigação de práticas predatórias ou danosas ao meio ambiente.

$\mathrm{O}$ avanço tecnológico de explotação dos recursos naturais transformou os oceanos em áreas de interesse econômico e estratégico; dessa forma, o território marítimo brasileiro, a chamada Amazônia Azul, compreenderia área superior a $3.600 \mathrm{~km}^{2}$, comparável com a área da floresta amazônica, a Amazônia Verde. 
A aprovação da Convenção das Nações Unidas sobre o Direito do Mar (CNUDM), em 1982, possibilita, no artigo 76, a expansão da margem continental para além das 200 milhas marítimas:

[...] para isto, o Estado costeiro deveria realizar uma pesquisa criteriosa e sugerir a fixação de limites, apresentando-os por meio de pleito a ser submetido à Comissão de Limites da Organização das Nações Unidas (ONU). (Martins; Carneiro, 2012, p.62).

Albuquerque (2006) assinala que, uma vez aprovado o pleito brasileiro, segundo o conceito de Plataforma Continental Jurídica Brasileira (PCJB), a inclusão de novas áreas ampliaria o direito do Brasil de explotar recursos, vivos ou não, e elevaria a área total do território nacional para $12.951 .766 \mathrm{~km}^{2}$ (um incremento de $4.436 .890 \mathrm{~km}^{2}$ o que equivale a $52 \%$ do total de terras emersas) (Martins; Carneiro, 2012). Essas áreas imensas, com enormes potenciais de exploração e geração de riquezas econômicas (minerais e biológicas), também necessitam de um grande contingente de profissionais capacitados para promover práticas exploratórias conscientes e, acima de tudo, entender as alterações do ambiente marinho em face das mudanças climáticas, com vistas à preservação da biodiversidade. $\mathrm{O}$ mesmo pode ser dito em relação à área florestal no domínio continental, com potencial biológico ainda bastante desconhecido e sofrendo ataques destrutivos que devastam grandes áreas de floresta tropical úmida. Essas ações, por sua vez, interferem nos padrões de circulação atmosférica no continente sul-americano e, de acordo com uma perspectiva sistêmica, introduzem alterações drásticas no regime pluviométrico de distintas regiões.

Outro grande desafio da atualidade, em que as Geociências aparecem como protagonista, é a busca por fontes alternativas de energia e, sobretudo, a chamada energia limpa. Embora ainda incipiente no Brasil, a exploração da energia eólica e solar aparece como alternativa francamente viável, sendo a primeira mais econômica. A energia das marés, ou energia maremotriz, gerada por meio do movimento das marés e explotada em alguns países, embora citada como de alto custo para instalação e manutenção e de rápida depreciação devido à água salgada, não deve ser descartada em face dos desafios da preservação ambiental.

Cabe ainda lembrar a gestão de recursos hídricos, assunto multidisciplinar, e que se coloca com grande prioridade, não só no Brasil, mas em todo o mundo. Os longos períodos de estiagem que temos observado levam à escassez de água para o consumo das populações, o que acarreta extração indiscriminada da água subterrânea. Poucos estudos têm sido dedicados aos processos de carga e descarga desses mananciais, bem como à preservação dos mesmos para que se possa usufruir de seus benefícios por longos períodos. Por outro lado, a gestão dos recursos hídricos requer uma visão integrada do sistema e das oscilações climáticas atuais dentro do contexto de competição entre uso da água para as populações e/ou irrigação e/ou geração de energia hidrelétrica. 
Os temas abordados aqui não esgotam as questões primordiais em que os profissionais das Geociências podem contribuir por intermédio de pesquisas ou como profissionais qualificados para atuar no mercado de trabalho, mas sinalizam a importância de se levar em consideração os conhecimentos advindos dessas áreas de pesquisa e incluir esses profissionais nos quadros dos organismos oficiais responsáveis por definir políticas públicas para os setores correspondentes.

\section{Desafios para o ensino de qualidade}

$\mathrm{Na}$ atualidade, é impensável descrever ou procurar entender qualquer fenômeno terrestre natural sem o auxílio de estatísticas, métodos numéricos e computacionais. Mesmo na Geologia, que já teve um apelo mais descritivo, encontra-se referência do uso da trigonometria em estudo de mineração, já no século XVI (Agricola, 1556; in Merriam, 2004). A Unesco reconhece como o marco inicial da hidrologia a obra de Pierre Perrault (De l'origine des fontaines, 1667) que relacionou quantitativamente a precipitação pluvial com as surgências (fonte) de águas.

Embora algumas áreas das Geociências sempre dependeram de medições e análise quantitativa de dados, outras tiveram desenvolvimento mais lento nesse sentido, mas, ao final, nenhum conhecimento geocientífico é produzido sem lançar mão da quantificação. Citando Lord Kelvin,

If you can not measure it, you can not improve it.

[...] when you cannot measure it, when you cannot express it in numbers, your knowledge is of a meagre and unsatisfactory kind; it may be the beginning of knowledge, but you have scarcely in your thoughts advanced to the state of Science, whatever the matter may be." [PLA, v.1, "Electrical Units of Measurement", 1883-05-03]

Além do mais, a integração das áreas do conhecimento leva os profissionais, obrigatoriamente, a se inteirar de outras metodologias, mais ou menos dependentes de métodos matemáticos ou de tecnologias mais sofisticadas. As leis da física permeiam todos os campos, portanto não há como dissociar as Geociências das Ciências Exatas. Essa percepção, entretanto, não é a mesma que muitos aspirantes aos cursos de terceiro grau têm em relação a algumas especializações das Ciências da Terra e, até mesmo, buscam aí um "refúgio" para não enfrentar disciplinas para as quais, muitas vezes, não receberam a formação adequada nos níveis básico e médio. O despreparo dos estudantes para acompanhar cursos de Exatas na Universidade é causa de desestímulo e frustração, como também é um dos fatores da alta evasão dos cursos nos quais essas matérias condicionam fortemente a progressão curricular. A lei do Ensino Médio enfatiza a relevância do domínio da linguagem matemática no Ensino Médio (ver análise de Pinto, 2017), mas a tônica deve ser dada desde o Ensino Fundamental, para que haja tempo suficiente para que o aluno absorva a linguagem matemática praticada no cotidiano e aquela que descreve os fenômenos físicos. Para isso é preciso desmitificar as Ciências Exatas e a integração com as Geociências que descrevem 
o meio em que o aluno está inserido (terra, mar e ar) e cujos fenômenos são vivenciados diariamente, o que pode representar práticas motivadoras e auxiliar na permanência na escola. Estudar o planeta Terra de maneira holística, considerando sua longa história de mudanças ambientais, processos e ciclos, ajuda a entender os impactos ambientais e riscos naturais. Os professores precisam ser capazes de desenvolver nos alunos uma compreensão profunda e duradoura dos conceitos científicos e estimular que os alunos construam habilidades e competências diretamente associadas à prática da ciência (Owen et al., 2004).

$\mathrm{O}$ ensino de nível superior também carece de atenção. $\mathrm{O}$ ensino das Geociências no Brasil continua ocorrendo em cursos que tem pouca comunicação entre si. Geologia, Geofísica, Meteorologia e Oceanografia têm currículos quase independentes com poucas intersecções. Pode-se imaginar que uma base inicial comum das Ciências da Terra poderia ser oferecida deixando a especialização para uma fase final. Será que assim seria possível uma formação integrada levando a uma visão de futuro, construtiva, realmente interdisciplinar? Será que novas técnicas de ensino, do tipo aprender fazendo, o uso de tecnologias da informação, entre outras, não permitiriam uma maior eficiência no ensino, com menos horas de ensino tradicional em salas de aula?

As experiências práticas, em laboratório ou campo, são atividades essenciais para que os alunos vivenciem as teorias que se deseja ensinar. Em Geologia, Geofísica, Meteorologia e Oceanografia, as atividades de campo são indispensáveis. Entretanto, por serem atividades que exigem aportes consideráveis de recursos financeiros, são por vezes minimizadas ou negligenciadas pelos gestores. A maioria dos cursos é ministrada por instituições públicas que continuamente enfrentam cortes de verbas. Nesse particular, o estímulo aos estudantes, por meio de bolsas para a participação em programas de Iniciação Científica, é extremamente importante, pois permite formação mais aprofundada e específica na utilização de certas metodologias, observações de campo, tratamento de dados e até mesmo no aperfeiçoamento de redação técnica. As entidades governamentais responsáveis por esses programas têm que zelar para que se possa mantê-los e ampliá-los. Os trabalhos práticos de conclusão de curso, adotados amplamente como parte da formação dos graduandos, seguem na mesma linha e são igualmente imprescindíveis.

A Resolução n.3 de 14.10.2010, que regulamenta o artigo 52 da LDB de 1996, ampliou as exigências para o credenciamento e recredenciamento do sistema federal de Ensino Superior, dentre elas, a proporção de um terço do corpo docente com titulação de mestrado ou doutorado. A norma trouxe um novo patamar para a qualidade do ensino nas universidades. Por outro lado, o sistema de avaliação dos programas de pós-graduação instituído pela Coordenação de Aperfeiçoamento de Pessoal de Nível Superior (Capes), a partir de sua reestruturação em 1995, alavancou, como consequência, a busca por pesquisas científicas de maior qualidade e mais competitivas no cenário internacional. 
Segundo o relatório de avaliação quadrienal Capes de 2017,

[...] as Geociências no Brasil tradicionalmente possuem um elevado patamar de consolidação e internacionalização, o que é registrado pelo intenso intercâmbio com instituições estrangeiras do hemisfério norte. Por estas razões, tem sido notável o fluxo de pesquisadores visitantes nos programas de excelência. Deve se ressaltar que muito dessas ações exercitadas pelos programas de excelência tem se realizado na forma de redes de pesquisa e, neste particular, os estudos de oceanografia e de mudanças climáticas, que envolvem a região amazônica, têm servido como laboratório para modelagens globais de alto impacto.

\section{Considerações finais}

O estudo das Geociências não está baseado em generalizações abstratas, mas se concentra em particularidades concretas da natureza; portanto, os conceitos são de muito mais fácil assimilação para os estudantes expostos a esses conteúdos. Para Bezzi (1999), os geocientistas e educadores das ciências da Terra têm a grande responsabilidade de transformar a educação em Geociências em um processo que deve ir além do mero ensino e aprendizagem dos fatos, leis e teorias; deve envolver a compreensão da natureza e suas relações com a sociedade.

King (2008) menciona estudos que revelam o interesse das crianças em grandes eventos da Terra, tanto do passado geológico como do presente e do futuro, assim como nas mudanças ambientais atuais que têm implicações diretas para o futuro da humanidade. Claramente, portanto, estratégias eficazes de ensino das ciências da Terra estarão relacionadas aos interesses desses alunos. A importância do uso de atividades como parte do ensino de Geociências foi enfatizada em diversos estudos sobre Educação em Geociências. Por exemplo, Chang (2001) observou que simulações em computador para resolução de problemas na sala de aula tendem a aumentar o envolvimento dos alunos no tema apresentado. Vê-se, portanto, que no mundo informatizado, tal como é o mundo moderno, é necessária a introdução de novas práticas de ensino, assim como mais atenção a instituições auxiliares do ensino, em outros locais de educação não formal, como exposições e museus interativos.

Num panorama mundial da Educação em Geociências, King (2008) verificou que as Geociências aparecem como uma pequena parte obrigatória de um currículo nacional de ciências. Assim que, em alguns países da Europa é parte de "ciências naturais" e geralmente ensinada por especialistas em biologia, ou química ou por professores de Ciências gerais. No Brasil, assim como no norte da Europa e Alemanha, é ensinado como parte integrante do conteúdo de geografia. Em sua análise concluiu que a Educação em Geociências progredirá mais eficazmente por intermédio da extensão da aprendizagem a todas as crianças e na orientação a professores quanto a efetiva implementação de novas iniciativas curriculares. 
O currículo de Geociências do Ensino Fundamental e Médio praticado no Brasil merece ser revisto e os professores encarregados de ministrar os conteúdos de Geociências precisam de formação mais específica. A modalidade do mestrado profissional em Geociências, a exemplo do que já é oferecido em algumas áreas em instituições do país, e voltado a professores do Ensino Fundamental e Médio, pode ser um caminho interessante para a difusão do conhecimento e a preparação de professores de ciências exatas ou biológicas que irão trabalhar diretamente com crianças e jovens nas escolas.

Nota

I Disponível em: <http://portal.inep.gov.br/pisa >.

\section{Referências}

ABBADI, Z. Security metrics: What can we measure. Open Web Application Security Project (Northern VA chapter), Apr. 2007, [online] URL: www.owasp.org/images/b/ b2/Security_Metics-_What_can_we_measure-_Zed_Abbadi.pdf. Acesso 01.out.2018.

ALBUQUERQUE, A. T. M. Bandeirantes das Longitudes Salgadas. In: CHAVES, P. de T. (Org.) O mar no espaço geográfico brasileiro. Brasília: Secretaria de Educação Básica, Ministério da Educação. 2006. p.19-24. (Coleção Explorando o Ensino).

BEZZI, A. What is this thing called geoscience? Epistemological dimensions elicited with the repertory grid and their implications for scientific literacy. Science Education, v.83, p.675-700, 1999.

BUENO, G. M. G.; FARIAS, S. A.; FERREIRA, L. H. Concepções de ensino de ciências no início do século XX: o olhar do educador alemão Georg Kerschensteiner. Ciência \& Educação, v.18, n.2, p.435-50, 2012.

CARNEIRO, C. D. R.; GONÇALVES, P. W. Earth System Science for undergraduate Geology and Geography courses, Campinas, Brazil. Terrae, v.7, n.1, p.29-40, 2010. Disponível em: <http://www.ige.unicamp.br/terrae/>.

CHANG, C. Y. Comparing the impacts of problem-based computer-assisted instruction and the direct interactive teaching method on student science achievement. Journal of Science Education and Technology, v.10, p.147-53, 2001.

CHASSOT, A. Parâmetros curriculares nacionais. Educação e Realidade, v.21, n.1, p.265-71, 1996.

CORDANI, U. G. Geosciences and development: The role of the earth sciences in a sustainable world. Ciência e Cultura, v.50, n.5, p.336-41, 1998.

DRUMMOND, C. N.; MARKIN, J. M. An analysis of the Bachelor of Science in Geology degree as offered in the United States. J. Geosc. Educ., v.56, n.2, p.113-19, 2008.

ENRICH, P. R.; HOLDREN, J. P. Impact of population growth. Science, v.171, p.1212-16, 1971.

GALlO, J.; Verrone, L. V. O que é Oceanografia. São Paulo: Brasiliense, 1993. p.115. (Col. Primeiros Passos). 
KING, C. Geoscience education: an overview. Studies in Science Education, v.44, p.187$222,2008$.

KRASILCHIK, M. Reformas e Realidade: o caso do ensino das ciências. São Paulo em Perspectiva, v.14, p. 85-93, 2000.

LIBÂNEO, J. C.; OLIVEIRA, J. F.; TOSCHI, M. S. Educação escolar: políticas, estrutura e organização. 10.ed. São Paulo: Cortez Editora, 2012.

MARTINS, J. R. S.; CARNEIRO, C. D. R. Plataforma continental jurídica, recursos do pré-sal e ensino de Geociências. Campinas, Terre, v.9, p.61-109, 2012. Disponível em: <http://www.ige.unicamp.br/terrae/V9/T_V9_A6.html>.

MERRIAM, D. F. The quantification of geology: from abacus to Pentium a chronicle of people, places, and phenomena. Earth-Science Reviews, v.67, p.55-89, 2004.

OWEN, J. C. et al. Design, implementation, and assessment of an Earth Systems Science course for secondary teachers. J. Geosc. Educ., v.52, n.4, p.379-85, 2004.

PINTO, A. H. A Base Nacional Comum Curricular e o Ensino de Matemática: flexibilização ou engessamento do currículo escolar. Bolema, v.31, n.59, p.1045-60, 2017.

SCAFETTA, N. The complex planetary synchronization structure of the solar system. Pattern Recognition in Physics. v.2, n.1, p.1-19, 2014. Disponível em: <http://dx.doi. org/10.5194/prp-2-1-2014>.

RESUMO - O ensino de Ciências foi incorporado aos currículos escolares somente no século XIX. No Brasil-Império o ensino das Ciências Naturais era incipiente, embora ciências já fizessem parte do currículo do ensino secundário desde a criação do Colégio Pedro II, em 1837. Os primeiros cursos de nível superior em Geociências (Geologia, Geofísica, Meteorologia e Oceanografia) começaram a ser criados somente a partir da segunda metade do século passado. Atualmente, há cursos nessas modalidades em todo o país, que enfrentam, contudo, sérios obstáculos para que o ensino evolua de acordo com as necessidades atuais do mercado de trabalho, e das tendências impostas pelo avanço tecnológico. Neste trabalho analisamos entraves e lacunas profissionais no Ensino Superior e destacamos algumas práticas educacionais capazes de melhorar o aprendizado, que poderiam ser universalizadas. É inadiável expandir a área de Geociências nos cursos de formação e capacitação de professores para a Educação Básica; o artigo reúne alguns fatores essenciais para atingir essa meta.

PALAVRAS-CHAVE : Geociências, Ensino Superior, Currículo,Educação Básica, Tecnologia.

ABSTRACT - The teaching of Science was incorporated into school curricula only in the nineteenth century. In Imperial Brazil, the teaching of the natural sciences was incipient, although, since the creation of the Colégio Pedro II in 1837, Science was already part of the curriculum of secondary education. But only in the second half of the last century the earliest courses in Geosciences (Geology, Geophysics, Meteorology, and Oceanography) began to be created. Currently, there are courses in these modalities across the country. However, there are serious obstacles for teaching to evolve in keeping with the current needs of the professional market and the trends imposed by tech- 
nological advances. In this work, we analyze obstacles and professional gaps in Higher Education, and highlight some educational practices capable of improving learning and which could be universalized. It is no longer possible to put off the expansion of the area of Geosciences in teacher training and qualification courses for Basic Education. This article brings together some essential factors to achieve this goal.

KEYWORDS : Geosciences, Higher education, Curriculum, Basic Education, Technology.

Marcia Ernesto é professora do Instituto de Astronomia, Geofísica e Ciências Atmosféricas da USP. @-mernesto@usp.br

Umberto G. Cordani é professor do Instituto de Geociências da USP. Foi diretor do Instituto de Estudos Avançados da USP (1994-1997). @- ucordani@usp.br

Celso dal Ré Carneiro é professor do Instituto de Geociências da Unicamp.

@ - cedrec@ige.unicamp.br

Maria Assunção F. da Silva Dias é professora do Instituto de Astronomia, Geofísica e Ciências Atmosféricas da USP. @- massuncao.dias@usp.br

Carlos Alberto Mendonça é professor do Instituto de Astronomia, Geofísica e Ciências Atmosféricas da USP. @- carlos.mendonca@iag.usp.br

Elisabete de Santis Braga é professora da Instituto Oceanográfico da USP.

@ - edsbraga@usp.br

Recebido em 15.9.2018 e aceito em 10.10.2018.

I, IV e V Instituto de Astronomia, Geofísica e Ciências Atmosféricas, Universidade de São Paulo, São Paulo, São Paulo, Brasil.

II Instituto de Geociências, Universidade de São Paulo, São Paulo, São Paulo, Brasil.

III Instituto de Geociências, Universidade Estadual de Campinas, Campinas, São Paulo, Brasil.

VI Instituto Oceanográfico, Universidade de São Paulo, São Paulo, São Paulo, Brasil. 
